extent and with a gas density of $20-100 \mathrm{~cm}^{-3}$ (ref. 26). 30 Dor is 200 times larger and 100-1,000 times less dense than the supernebula in NGC5253, and its exciting star cluster R136 is 10-100 times less massive than the cluster in NGC5253 (ref. 27). The escape velocity for 30 Dor is less than the thermal sound speed of $10 \mathrm{~km} \mathrm{~s}^{-1}$ so its gas motions are determined by winds and turbulence rather than by gravity $^{28}$. How the supernebula in NGC5253 will free itself from 'gravitational bondage' and evolve into an extended, diffuse nebula like 30 Dor will probably depend on the evolution of its underlying star cluster.

Received 27 November 2002; accepted 28 April 2003; doi:10.1038/nature01689.

1. Turner, J. L., Beck, S. C. \& Ho, P. T. P. The radio supernebula in NGC5253. Astrophys. J. 532, L109-L112 (2000)

2. Meier, D. S., Turner, J. L. \& Beck, S. C. Molecular gas and star formation in NGC5253 revisited. Astron. J. 124, 877-885 (2002).

3. Beck, S. C., Turner, J. L., Ho, P. T. P., Kelly, D. \& Lacy, J. H. The central star cluster of the star-forming dwarf galaxy NGC5253. Astrophys. J. 457, 610-615 (1996).

4. Turner, J. L., Ho, P. T. P. \& Beck, S. C. The radio properties of NGC5253 and its unusual HII regions. Astron. J. 116, 1212-1220 (1998).

5. Gorjian, V., Turner, J. L. \& Beck, S. C. Infrared emission from the radio supernebula in NGC5253: A proto-globular cluster? Astrophys. J. 554, L29-L32 (2001).

6. Caldwell, N. \& Phillips, M. M. Star formation in NGC5253. Astrophys. J. 338, 789-803 (1989).

7. Gorjian, V. WFPC2 imaging of the starburst galaxy NGC5253. Astron. J. 1120, 1886-1893 (1996).

8. Calzetti, D. et al. Dust and recent star formation in the core of NGC5253. Astron. J. 114, 1834-1849 (1997).

9. Tremonti, C. A., Calzetti, D., Leitherer, C. \& Heckman, T. M. Star formation in the field and clusters of NGC5253. Astrophys. J. 555, 322-337 (2001).

10. McLean, I. S. et al. Performance and results with the NIRSPEC echelle spectrograph on the Keck II Telescope. Proc. SPIE 4008, 1048-1055 (2000).

11. Kawara, K., Nishida, M. \& Phillips, M. M. Brackett alpha and gamma observations of starburst and Seyfert galaxies. Astrophys. J. 337, 230-235 (1989)

12. Storey, P. J. \& Hummer, D. G. Recombination line intensities for hydrogenic atoms. IV. Total recombination coefficients and machine-readable tables for $Z=1$ to 8. Mon. Not.R. Astron. Soc. 272, $41-48$ (1995).

13. Walsh, J. R. \& Roy, J.-R. Optical spectroscopic and abundance mapping of the amorphous galaxy NGC5253. Mon. Not. R. Astron. Soc. 239, 297-324 (1989).

14. Rieke, G. H. \& Lebofsky, M. J. The interstellar extinction law for 1 to 13 microns. Astrophys. J. 288, 618-621 (1985).

15. Mohan, N. R., Anantharamaiah, K. R. \& Goss, W. M. Very Large Array observations of the H92 $\alpha$ line from NGC5253 and Henize 2-10: Ionized gas around super star clusters. Astrophys. J. 557, 659-670 (2001).

16. Martin, C. L. \& Kennicutt, R. C. Jr Soft X-ray emission from NGC5253 and the ionized interstellar medium. Astrophys. J. 447, 171-183 (1995).

17. De Pree, C. G., Wilner, D. J., Goss, W. M., Welch, W. J. \& McGrath, E. Ultracompact HII regions in W49N at 500 AU scales: Shells, winds, and the water maser source. Astrophys. J. 540, 308-315 (2000)

18. Chu, Y. H. Ring nebulae around massive stars throughout the HR diagram. In IAU Symposium 212 (eds van der Hucht, K. A., Herrero, A. \& Esteban, C.) (in the press).

19. Wood, D. O. S. \& Churchwell, E. Massive stars embedded in molecular clouds: Their population and distribution in the Galaxy. Astrophys. J. 340, 265-272 (1989).

20. Kunth, D. et al. HST study of Lyman-alpha emission in star-forming galaxies: the effect of neutral gas flows. Astron. Astrophys. 334, 11-20 (1998).

21. Tenorio-Tagle, G., Silich, S. A., Kunth, D., Terlevich, E. \& Terlevich, R. The evolution of superbubbles and the detection of Lya in star-forming galaxies. Mon. Not. R. Astron. Soc. 309, 332-342 (1999).

22. Meier, D. L. \& Terlevich, R. Extragalactic HII regions in the UV. Implications for primeval galaxies. Astrophys. J. 246, L109-L113 (1981).

23. Hartmann, L., Huchra, J. P. \& Geller, M. J. How to find galaxies at high redshift. Astrophys. J. 287, 487-491 (1984).

24. Steidel, C. C., Giavalisco, M., Pettini, M., Dickinson, M. \& Adelberger, K. L. Spectroscopic confirmation of a population of normal star-forming galaxies at redshifts z $>3$. Astrophys. J. 462, L17-L21 (1996).

25. Walborn, N. R. \& Barbá, R. H. in New Views of the Magellanic Clouds (eds Chu, Y.-H., Suntzeff, N Hesser, J. \& Bohlender, D.) 213 (Astronomical Society of the Pacific, San Francisco, 1999).

26. Mills, B. Y., Turtle, A. J. \& Watkinson, A. A radio model of the 30 Doradus region. Mon. Not. R. Astron. Soc. 185, 263-276 (1978).

27. Kennicutt, R. C. Jr \& Chu, Y.-H. Giant HII regions and the rormation of populous clusters. Astron. J. 95, 720-730 (1988).

28. Melnick, J., Tenorio-Tagle, G. \& Terlevich, R. Supersonic gas motion in extragalactic HII regions. Mon Not. R. Astron. Soc. 302, 677-683 (1999).

29. Moorwood, A. F. M. \& Glass, I. S. Infrared emission and star formation in NGC5253. Astron. Astrophys. 115, 84-89 (1982).

Acknowledgements We thank T. Glassman and E. Greisen for assistance with the data and M. Jura for discussions. This research is supported by the US National Science Foundation, the Israel Academy Center for Multi-Wavelength Astronomy, and the Laboratory of Astronomical Imaging at the University of Illinois.

Competing interests statement The authors declare that they have no competing financial interests.

Correspondence and requests for materials should be addressed to J.T. (turner@astro.ucla.edu).

\section{A strong decrease in Saturn's equatorial jet at cloud level}

\author{
A. Sánchez-Lavega ${ }^{\star}$, S. Pérez-Hoyos ${ }^{\star}$, J. F. Rojas $\dagger$, R. Hueso* \\ \& R. G. French $\ddagger$
}

* Departamento Física Aplicada I, Escuela Superior de Ingenieros, Universidad del País Vasco, Alameda Urquijo s/n, 48013 Bilbao, Spain

$\dagger$ Departamento Física Aplicada I, EUITI, Universidad del País Vasco,

Plaza Casilla s/n, 48013 Bilbao, Spain

$\ddagger$ Department of Astronomy, Wellesley College, Wellesley, Massachusetts 02481, USA

The atmospheres of the giant planets Jupiter and Saturn have a puzzling system of zonal (east-west) winds alternating in latitude, with the broad and intense equatorial jets on Saturn having been observed previously to reach a velocity of about $470 \mathrm{~m} \mathrm{~s}^{-1}$ at cloud level ${ }^{1}$. Globally, the location and intensity of Jupiter's jets are stable in time to within about ten per cent ${ }^{2,3}$, but little is known about the stability of Saturn's jet system. The longterm behaviour of these winds is an important discriminator between models for giant-planet circulations ${ }^{4-9}$. Here we report that Saturn's winds show a large drop in the velocity of the equatorial jet of about $200 \mathrm{~m} \mathrm{~s}^{-1}$ from 1996 to 2002. By contrast, the other measured jets (primarily in the southern hemisphere) appear stable when compared to the Voyager wind profile of 1980-81.

The first precise measurements of Saturn's zonal wind velocity profile were performed during the Voyager encounters in 1980-81 (refs 10, 11). Previous wind measurements, obtained by tracking individual 'spots' in the atmosphere, are scarce, but are in general agreement with the Voyager data ${ }^{12}$. Cloud motions measured on Voyager images revealed a strong and broad equatorial jet (peak velocity $\sim 470 \mathrm{~m} \mathrm{~s}^{-1}$, spanning planetographic latitudes $\pm 40^{\circ}$ ), twice as broad and four times more intense than that of Jupiter. The equator is also the place where infrequent but violent eruptions (the 'Great White Spots') occur ${ }^{13-15}$. As Saturn has a large obliquity of $26.7^{\circ}$, and the shadow of the rings produces strong insolation changes in the equatorial region, induced effects during Saturn's year (29.4 terrestrial years) could be expected at cloud level where the winds are measured ${ }^{16}$. The zonal jet measurements rely on the detection and tracking of cloud features, and because of the lower contrast, size and number of such features in Saturn than in Jupiter, this can only be performed at present using the Hubble Space Telescope (HST). The Voyager data were obtained during the northern hemisphere spring (1980-81), and HST high-quality images of Saturn are available for the period 1994-2002, during Saturn's southern hemisphere spring and early summer. Both epochs are well placed to look for long-term changes.

The HST images analysed here were obtained between 1996 and 2002 (3-6 days per year) using the Wide Field Planetary Camera 2 (WFPC2) at its maximum spatial resolution. A series of filters from 255 to $1,042 \mathrm{~nm}$ were used, including at times a filter isolating the 890-nm methane absorption band (dates and filters for each campaign are given in Supplementary Information). In total, about 100 images were selected, navigated on the planetary limb and photometrically calibrated. Images obtained at wavelengths of $439 \mathrm{~nm}, 675 \mathrm{~nm}, 814 \mathrm{~nm}$ and $890 \mathrm{~nm}$ showed the clouds to have high contrast (enhanced by an 'unsharp masking' processing), and were used for target identification and tracking. Image pairs or triplets obtained with the same filter, separated by a temporal interval ranging from 0.5 to 8 days, were used for the tracking. Following the ring-plane crossing epoch in 1995, Saturn's orbital obliquity and ring projection limited our visibility of the northern hemisphere, so most features were observed from latitudes $+30^{\circ}$ to 
$-90^{\circ}$. A total of 343 individual features (most with sizes ranging from $\sim 500$ to $2,000 \mathrm{~km}$ ) were tracked. A sample of Saturn's cloud features observed at different latitudes and with different filters, and their tracked motions in the equator, are shown in Fig. 1.

In Fig. 2 we present our 1996-2002 measurements, and compare them to the Voyager wind profile ${ }^{11}$. We also include individual spot velocities obtained during 1994-97 from ground-based telescopes ${ }^{17}$ (full wind speed data are available as Supplementary Information). First, the most evident and unexpected result is the truncation of the equatorial eastward jet to maximum velocities of $\sim 275 \mathrm{~m} \mathrm{~s}^{-1}$, a drop of $\sim 200 \mathrm{~m} \mathrm{~s}^{-1}$ relative to the Voyager value. The deviation from the Voyager profile took place equatorward of latitudes $\pm 17^{\circ}$, although the jet itself is slightly broader, starting to change at latitudes $\pm 25^{\circ}$. Second, there are no apparent changes in the other southern eastward and westward jets, even in the 'ribbon' wave jet at latitude $+47^{\circ}$ re-observed during 1994-96 (ref. 18). Last, we confirm the existence of a recently discovered south polar jet at $-73^{\circ}$ latitude $^{19}$, making Saturn's zonal jet system highly symmetric.

The HST profile is plotted using features measured with all filters and over the full time period indicated above. We do not find any dependence of wind velocities with wavelength, nor with time, during the observing period. The peak truncation has persisted since $\sim 1994$. The velocity dispersion seen in the equatorial region from $+5^{\circ}$ to $-17^{\circ}$ (amounting in some places up to $\sim 75 \mathrm{~m} \mathrm{~s}^{-1}$ ) is probably real, and reflects intense local motions related to the disturbances in the equatorial area (also observed one month after the 1990 storm outbreak ${ }^{15}$ ). A detailed analysis of the equatorial cloud morphology showed that this period was characterized by the development of large-scale features $(\sim 20,000 \mathrm{~km}$ in zonal extent) with abundant internal structure (Fig. 1). This differs from the Voyager era, when the most representative cloud features were series of bright plume-like structures ${ }^{11}$. However, between both periods, 1980-81 and 1996-2002, a large storm erupted at $+5^{\circ}$ in 1990 (refs 13-15), disrupting the equatorial region cloud morphology from latitudes $+22^{\circ}$ to $-5^{\circ}$, and extending its activity during 1991 (ref. 20). It was followed by another intense storm centred at $+10^{\circ}$ in 1994 (ref. 21), and by subsequent similar features during 1995-97 (ref. 17).

In order to determine the altitude of the features we see, we have carried out a radiative transfer analysis of the wavelength dependence of the reflectivity across selected latitudes from the equator to the south pole (from limb to limb), using the 'doublingadding technique'18,19,22 . At $-10^{\circ}$, where the main jet speed velocity changes are seen, the model that best fits the reflectivity has a high stratospheric haze (altitude between 1 and 10 mbar), and below it, a thick layer of particles (haze or cloud) with optical depth $\tau=10$ located between 40 and 300 mbar. Deeper (1.8 bar), a putative ammonia cloud is placed. But the features that we see (that is, the cloud tops, where $\tau \approx 1-2$ ) are located in the intermediate thick haze, nominally at pressures $\sim 100 \mathrm{mbar}$, close to the tropopause level ${ }^{23,24}$. According to cloud models of the equatorial region at the Voyager epoch ${ }^{25}$ and before the development of the 1990 storm $^{26}$, the equatorial haze layer reached $\tau=1$ in the level $\sim 300$ mbar. Our analysis of the 1990 storm placed its cloud tops at $200 \mathrm{mbar}$ (ref. 22).

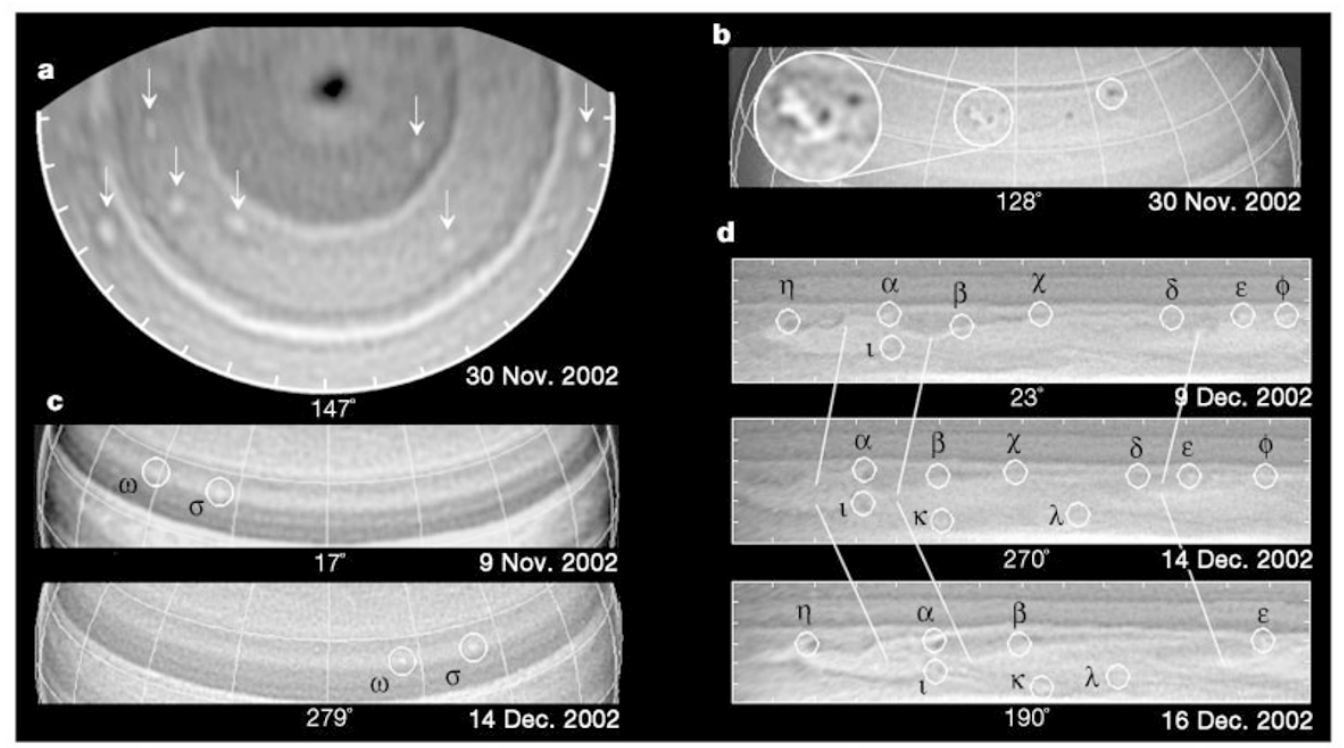

Figure 1 Images of different latitudes of Saturn, showing a representative variety of cloud features tracked to measure the winds. The HST WFPC2 images were obtained at different wavelengths, and in some cases their motions are indicated. The spatial resolution of the camera (PC mode) was $0.0455 \mathrm{arcsec}$ pixel ${ }^{-1}\left(\sim 260 \mathrm{~km} \mathrm{pixel}^{-1}\right.$ at Saturn). a, Polar projection. The pole is centred on the dark cap; the arrows mark the bright spots (at a wavelength of $890 \mathrm{~nm}$ ) at latitudes ranging from $-50^{\circ}$ to $-90^{\circ}$ (each tick mark represents $10^{\circ}$ in longitudinal extent). $\mathbf{b}$, Temperate latitudes at a wavelength of $675 \mathrm{~nm}$ (the full latitude circles shown are $-35^{\circ}$ and $-50^{\circ}$ ). The image shows regularly observed mid-latitude dark spots together with a bright spot (shown magnified in the inset to reveal its structure). c, Pair of images showing the zonal translation of two bright features at latitude $-29^{\circ}$ and at a wavelength of $439 \mathrm{~nm}$. The full latitude circles shown are $-17^{\circ}$ and $-33^{\circ}$ (note the change in central meridian longitude between the two dates). d, Cylindrical maps of the equator (from $0^{\circ}$ to $-30^{\circ}$ ), showing a variety of features at a wavelength of $890 \mathrm{~nm}$ and their zonal translations (note again the changing longitude of the central meridian for each day). Separations between tick marks are $10^{\circ}$ in longitude and $5^{\circ}$ in latitude. The three white lines identify typical large-scale equatorial features in the three maps. In $\mathbf{b}-\mathbf{d}$, south is up and east is to the left. Longitudes in the central meridian are based on the System III radio rotation period, and latitudes are planetographic ${ }^{11}$. The longitudinal translation of each feature in $\mathbf{c}$ and $\mathbf{d}$ was converted to drift rate (degrees per day) and then to zonal velocity (see Fig. 2). If the features shown in d (first panel) had moved at the Voyager zonal velocities, they would be about $40^{\circ}$ to the left of their marked positions in the second panel. This is substantially greater than the measurement uncertainty. 
Thus, within the model uncertainties, the equatorial features we see in 1996-2002 are slightly higher than in 1980-81, probably by no more than a scale height $(\sim 36 \mathrm{~km})$.

The abrupt truncation of the eastward equatorial jet could be the result of a true temporal change in the general circulation due to unknown dynamical processes, or an indirect effect. One possibility is that the cloud systems in 1996-2002, placed at a higher altitude than in 1980-81, moved more slowly, according to the expected decrease in altitude of zonal winds measured in other latitudes ${ }^{23}$. Another possibility is that wave or disturbance propagation at cloud level in the equator, relative to the mean zonal flow, is affecting our velocity measurements. However, the historical large-scale equatorial storms (in 1876, 1933 and 1990) moved with speeds $\sim 400 \mathrm{~ms}^{-1}$ (refs 11,20), close to the Voyager profile at the central latitude of the storms, indicating that such an effect was not present at least during the initial phase of the storm development.

The picture that emerges from our 1996-2002 measurements, when compared to the Voyager data for 1980-81, is that Saturn's equatorial dynamics and jet structure show high variability. It is the region where most of the large-scale storms form on the planet, and the place where the jet wind speed has changed by $42 \%$, as reported here. Such a large wind speed variability has not been detected in Jupiter, where many more observations on jet stability are avail-

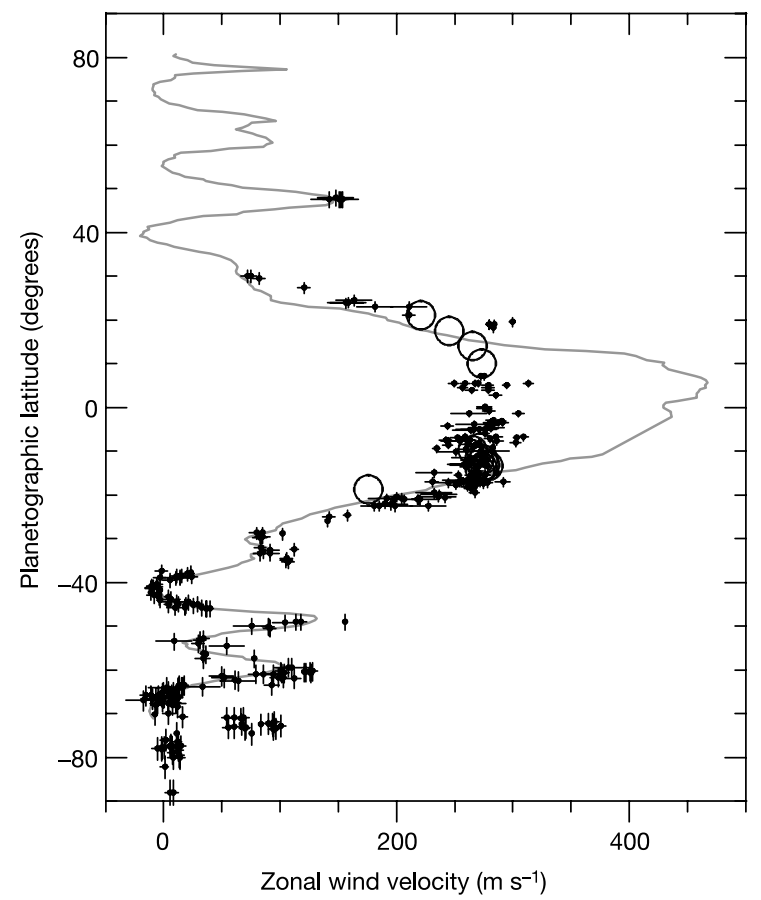

Figure 2 Saturn's zonal (east-west) wind velocity profile at cloud level as a function of latitude. Data are shown for two different epochs (1980-81 and 1995-2002). The continuous line shows the averaged Voyager 1 and 2 profiles, as measured during 1980-81 (ref. 11). The large open circles represent individual measurements obtained from 1995 to 1997 by tracking large features using ground-based telescopes ${ }^{17}$. The small black dots are the individual velocity measurements obtained between 1996 and 2002 using HST images as reported in the text, with the error for each individual measurement added as a thin line. The wind speeds are determined relative to the radio rotation period of $10 \mathrm{~h} 39 \mathrm{~min} 24 \mathrm{~s}$, assumed to be that of the interior. The main uncertainties in these data arise from image navigation (planetary limb fit), target misidentification, and cursor pointing on the feature. The errors in latitude positioning range from $\pm 0.5^{\circ}$ in the equator to $\pm 1.5^{\circ}$ at the poles. Wind speed precision is of the order of target size/tracking time; at our typical target size $(500-2,000 \mathrm{~km}$ ) and tracking times (minimum $0.5 \mathrm{~d}$, maximum $60 \mathrm{~d}$ ), this gives values ranging from $\pm 1 \mathrm{~m} \mathrm{~s}^{-1}$ to $\pm 15 \mathrm{~m} \mathrm{~s}^{-1}$ (see Supplementary Information for the full data for each point used in this figure). able ${ }^{2,3}$. Outside the equator, our observations indicate that Saturn's jets appear to be stable in time. Compared to Jupiter, Saturn has fewer jets overall, but they show higher north-south symmetry in latitude and in speed intensity. Saturn receives less sunlight and releases less internal heat than Jupiter, but the sunlight source is more variable owing to the planet's obliquity. For example, the polar areas each alternately fall into darkness during the course of a half Saturn year, and the equator suffers the ring-shadowing insolation variability. All these differences between the planets need to be considered when developing future general circulation models for the jet origin in the giant planets.

Received 5 February; accepted 11 April 2003; doi:10.1038/nature01653.

1. Ingersoll, A. P. Atmospheric dynamics of the outer planets. Science 248, 308-315 (1990).

2. García-Melendo, E. \& Sánchez-Lavega, A. A study of the stability of Jovian zonal winds from HST images: 1995-2000. Icarus 152, 316-330 (2001).

3. Porco, C. C. et al. Cassini imaging of Jupiter's atmosphere, satellites and rings. Science 299, 1541-1547 (2003)

4. Williams, G. P. Jovian and comparative atmospheric modeling. Adv. Geophys. A 28, 381-429 (1985).

5. Cho, J. Y. K. \& Polvani, L. M. The morphogenesis of bands and zonal winds in the atmospheres on the giant outer planets. Science 273, 335-337 (1996)

6. Busse, F. H. A simple model of convection in the Jovian atmosphere. Icarus 30, 255-260 (1976).

7. Ingersoll, A. P. \& Pollard, D. Motion in the interiors and atmospheres of Jupiter and Saturn: Scale analysis, anelastic equations, barotropic stability criterion. Icarus 52, 62-80 (1982).

8. Zhang, K. K. \& Schubert, G. Teleconvection: Remotely driven thermal convection in rotating stratified spherical layers. Science 290, 1944-1947 (2000).

9. Yano, J.-I., Talagrand, O. \& Drossart, P. Atmospheric zonal jets in the atmospheres of the giant planets simulated by an idealized two-dimensional deep-fluid model. Nature 421, 36 (2003).

10. Ingersoll, A. P., Beebe, R. F., Conrath, B. J. \& Hunt, G. E. in Saturn (eds Gehrels, T. \& Matthews, M. S.) 195-238 (Univ. Arizona Press, Tucson, 1984).

11. Sánchez-Lavega, A., Rojas, J. F. \& Sada, P. V. Saturn's zonal winds at cloud level. Icarus 147, 405-420 (2000).

12. Sánchez-Lavega, A. Motions in Saturn's atmosphere: Observations before Voyager encounters. Icarus 49, 1-16 (1982).

13. Sánchez-Lavega, A. et al. The Great White Spot and disturbances in Saturn's equatorial atmosphere during 1990. Nature 353, 397-401 (1991).

14. Beebe, R. F., Barnet, C., Sada, P. V. \& Murrell, A. S. The onset and growth of the 1990 equatorial disturbance on Saturn. Icarus 95, 163-172 (1992).

15. Barnet, C. D., Westphal, J. A., Beebe, R. F. \& Huber, L. F. Hubble Space Telescope observations of the 1990 equatorial disturbance on Saturn: Zonal winds and central meridian albedos. Icarus 100, 499-511 (1992).

16. Barnet, C. D., Beebe, R. F. \& Conrath, B. J. A seasonal radiative-dynamic model of Saturn's troposphere. Icarus 98, 94-107 (1992).

17. Sánchez-Lavega, A., Lecacheux, J., Colas, F., Rojas, J. F. \& Gomez, J. M. Discrete cloud activity in Saturn's equator during 1995, 1996 and 1997. Planet. Space Sci. 47, 1277-1283 (1999).

18. Sánchez-Lavega, A. Observations of Saturn's ribbon wave 14 years after its discovery. Icarus 158, 272-275 (2002).

19. Sánchez-Lavega, A., Pérez-Hoyos, S., Acarreta, J. R. \& French, R. G. No hexagonal wave around Saturn's southern pole. Icarus 160, 216-219 (2002).

20. Sánchez-Lavega, A., Lecacheux, J., Colas, F. \& Laques, P. Temporal behavior of cloud morphologies and motions in Saturn's atmosphere. J. Geophys. Res. 98, 18857-18872 (1993).

21. Sánchez-Lavega, A. et al. Large-scale storms in Saturn's atmosphere during 1994. Science 271, 631-634 (1996).

22. Acarreta, J. R. \& Sánchez-Lavega, A. Vertical cloud structure in Saturn's 1990 equatorial storm. Icarus 137, 24-33 (1999).

23. Conrath, B. J. \& Pirraglia, J. A. Thermal structure of Saturn from Voyager infrared measurements: Implications for atmospheric dynamics. Icarus 53, 286-292 (1983).

24. Lindal, G. F., Sweetnam, D. N. \& Eshleman, V. R. The atmosphere of Saturn: An analysis of the Voyager radio occultation measurements. Astron. J. 90, 1136-1146 (1985).

25. Tomasko, M. G., West, R. A., Orton, G. S. \& Tejfel, V. G. in Saturn (eds Gehrels, T. \& Matthews, M. S.) 150-194 (Univ. Arizona Press, Tucson, 1994).

26. Karkoschka, E. \& Tomasko, M. G. Saturn's upper troposphere 1986-1989. Icarus 97, 161-181 (1992)

Supplementary Information accompanies the paper on www.nature.com/nature.

Acknowledgements We thank M. Flasar for suggestions. The NASA/ESA Hubble Space Telescope is operated by AURA under NASA contract. A.S.-L., S.P.-H. and J.F.R. were supported by the MCYT Plan Nacional de Astronomía y Astrofísica and Grupos UPV. S.P.-H. acknowledges a PhD fellowship from the Spanish MECD, and R.H. a post-doctoral fellowship from Gobierno Vasco. R.G.F. was supported in part by NASA's Planetary Geology and Geophysics Program and an STSCI grant.

Competing interests statement The authors declare that they have no competing financial interests.

Correspondence and requests for materials should be addressed to A.S.-L.

(wupsalaa@bi.ehu.es). 